\title{
Washing operation of a road surface washing mechanism for decontaminating radioactive substances
}

\author{
Mitsuru Endo ${ }^{*}$, Mai Endo and Takao Kakizaki
}

\begin{abstract}
The Great East Japan Earthquake that occurred on March 11, 2011 resulted in the explosion of the TEPCO Fukushima 1st Nuclear Power Plant and the global dispersion of a large quantity of radioactive substances. A high radiation dose was particularly recorded in Fukushima prefecture several weeks after the accident, although the level is presently sufficiently low. However, considering that the adverse effects of low but extended exposure to radiation are yet to be negated, there is the urgent need for further decontamination. In our study, we focused on the efficient

decontamination of radioactive substances in residential areas, for which we propose a high-pressure water jet system for washing road surfaces. The system differs from conventional systems of its type that were initially designed for use in the immediate environment of the nuclear reactors of the TEPCO Fukushima 1st Nuclear Power Plant. The proposed system consists of multiple washing, transporter, and server robots. The washing robots decontaminate the road surface using high-pressure water jets and are transported between washed and unwashed areas by the transporter robots. The server robots supply the water used for washing and absorb the polluted water together with ground dust. In this paper, we describe the concept of the system and present the results of decontamination experiments. Particular attention is given to the washing robot and its mechanism and control method. The results of the integration of the washing robot in an experimental system confirmed the feasibility of the proposed system.
\end{abstract}

Keywords: Decontamination of radioactive substances; Multiple robot coordination; Water jet; Fukushima

\section{Background}

The Pacific side of Tohoku region was heavily damaged by The Great East Japan Earthquake that occurred on March 11, 2011, which resulted in the explosion of the TEPCO Fukushima 1st Nuclear Power Plant and the global dispersion of a large quantity of radioactive substances. A high radiation dose was recorded several weeks after the accident, especially in Fukushima prefecture. The radiation dose has, however, become sufficiently low and there has been no immediate increase in health problems caused by radioactive substances. Nevertheless, the dose of radioactive substances with longer half-lives remains higher than ever before.

Immediately after the accident, the government announced plans to decontaminate all areas with high

*Correspondence: m_endo@mech.ce.nihon-u.ac.jp

Department of Mechanical Engineering, Nihon University, Koriyama, 963-8642 Fukushima, Japan radiation doses, namely, more than $1 \mathrm{mSv} /$ year, or 0.23 $\mu \mathrm{Sv} / \mathrm{hour}$. Based on this standard, the government developed plans and guidelines for long-term activities. Accordingly, since 2011, both decontamination and decommissioning have been ongoing in the immediate surroundings of the nuclear reactors of the TEPCO Fukushima 1st Nuclear Power Plant. Moreover, since 2012, the decontamination of the cities in Fukushima prefecture has been vigorously pursued.

In the city of Koriyama, which is located at almost the center of Fukushima, the decontamination of about 100 houses was completed between June and August 2012. Based on the results of the decontamination, the city of Koriyama announced the objective of decontaminating 25,000 houses before the end of 2012. However, the number of houses that were eventually decontaminated in 2012 was less than 1,000. This failure to achieve the optimistic target of the city revealed the difficulty of

\section{望 Springer}

(c) 2014 Endo et al.; licensee Springer. This is an Open Access article distributed under the terms of the Creative Commons Attribution License (http://creativecommons.org/licenses/by/2.0), which permits unrestricted use, distribution, and reproduction in any medium, provided the original work is properly credited. 
decontamination. It clearly showed that the decontamination as announced by the city was difficult to achieve without technological innovation.

To overcome the difficulties of decontamination, there have been active attempts to develop new technology for managing the aftermaths of the accident at the TEPCO Fukushima 1st Nuclear Power Plant. In 2013, the government and Fukushima prefecture inaugurated the International Research Institute for Nuclear Decommissioning and the Fukushima Decommissioning and Decontamination Robot Technology Meeting, among other similar establishments. The purpose of these establishments was the acceleration of the decontamination and decommissioning activities around the TEPCO Fukushima 1st Nuclear Power Plant . However, their technological developments were not concerned with the living conditions of the general citizenry but the power plant.

Studies on the long-term health hazards of people living near nuclear accident sites have been limited to the Chernobyl Nuclear Power Plant, and there are quite a few examples. However, such study focusing on the situation in Fukushima, which has a lower radiation dose than Chernobyl, is unprecedented. Hence, it is difficult to discuss the health hazards in Fukushima. Moreover, considering that the adverse effects of very low but extended exposure to radiation have not been negated, there is an urgent need for technological development for the radioactive decontamination of residential areas.

\section{Robotic system for decontamination and decommissioning}

Shortly after the accident at the TEPCO Fukushima 1st Nuclear Power Plant, large manufacturers, government organizations, and researchers proposed several decontamination systems that utilize robot technology. The robotic systems were designed to clear the rubble heaps produced by the explosion; explore the area, which had a very high radiation dose; and decontaminate the surroundings of the nuclear reactors. Most of them were produced for practical application [1].

For example, Hitachi proposed the decontamination robotic system named "Arounder". The system consisted of a mobile manipulator with a high-pressure washing system on its end-effector and a mobile robot with a compressor for high-pressure washing and vacuuming. Toshiba proposed a system that was similar to that of Hitachi. It comprised a dry ice blast system that was operated alternately with a high-pressure washing system. Mitsubishi also proposed a dual-arm robotic system that could be used for both decontamination and the handling and cutting of rubble by changing the end-effector.

Each of these manufacturers proposed other types of robotic systems for the TEPCO Fukushima 1st Nuclear Power Plant. Hitachi proposed a tele-operated dual-arm robot for decommissioning nuclear reactors. Mitsubishi and Honda/AIST developed a tele-operated robot for work at elevated locations. Mitsubishi also developed a robot that could move on both ground and water for investigating water leaks on brown reactors.

Regarding researchers, Koyanagi proposed robots named Quince, Quince II, and Sakura, which could be used instead of humans for investigating the condition of the exploded reactors and the high radiation dose, among other purposes [2]. The robotic systems had actually been previously developed for search and rescue operations on disaster sites. Shortly after the Fukushima power plant accident, they were remodeled for investigation of the nuclear reactors. Hirose also proposed a tendon-driven mobile manipulator that could operate in an environment containing complicated pipes, such as inside the building of a nuclear reactor [3]. These robotic systems are feasible for an environment with a very high radiation dose, such as inside the building of a nuclear reactor or inside the reactor itself, and are expected to find major applications in the near future. However, they cannot be easily applied in a residential area because they were primarily designed for the decontamination and/or decommissioning of the TEPCO Fukushima 1st Nuclear Power Plant.

In the present study, we focused on a robotic system that could be used for decontamination activities in residential areas, such as washing of roads. The proposed system, which comprises multiple robots, was designed for continuous and efficient decontamination.

\section{Decontamination of a residential area Decontamination techniques}

The proposed system decontaminates a road by washing using high-pressure water. High-pressure water washing, as a standard method for decontaminating roads, is basically recommended in the guidelines of the Ministry of the Environment of Japan. The decontamination efficiency of this method is about $60 \%$, which is relatively low. It, however, enables decontamination by commercially available machines.

To achieve more efficient decontamination, large manufacturers, government organizations, and researchers have proposed novel methods. The methods can be classified into three categories, namely,

(1) removal of radioactive substances by grinding them against the decontaminated surface,

(2) liberation and removal of the radioactive substances from the decontaminated surface,

(3) washing the decontaminated surface.

The use of dry ice blast, shot blast, and other massive collisions, as well as removal of the surface soil, are examples of methods involving grinding against the decontami- 
nated surface. The use of a surfactant or isolating chemical coats are examples of methods involving liberation and removal from the decontaminated surface, whereas high-pressure and super-high-pressure washing are examples of methods involving washing of the decontaminated surface.

The efficiencies of these methods are about $90 \%$, which is higher than that of the basic method recommended by the Ministry of the Environment. However, these methods generally require large equipment such as a pumper and vacuum car, and other special devices. Even when large equipment is not required, there is the need for special chemicals, which makes the methods unsuitable for residential areas.

The guidelines published by the Ministry of the Environment suggest the use of the high-efficiency methods mentioned above when the radiation dose cannot be sufficiently reduced by high-pressure water decontamination. Shot blast, super-high-pressure water, and grinder processing are particularly suggested. However, as mentioned above, these methods require large equipment. Thus, even when the radiation dose cannot be sufficiently reduced by high-pressure water, decontamination workers leave the situation like that. It is therefore expedient to explore means of sufficiently reducing the radiation dose using commercially available high-pressure washing machines.

\section{Efficient decontamination using a commercially available high-pressure washing machine}

We propose a method based on fluid dynamics for decontamination using a commercially available highpressure washing machine. By this method, the highpressure washing machine suggested by the Ministry of the Environment can be used to reduce the radiation dose by about $90 \%$.

The guidelines of the Ministry of the Environment describe the normal use of the machine for decontamination. This involves holding the washing nozzle near the waist, blasting the water against the target such as the road or wall, and washing the entire surface. In the proposed method, however, the nozzle is not held near the waist but very close to the target. To be specific, the distance between the nozzle and the target is controlled at about $0.01 \mathrm{~m}$. Regardless of the water pressure, as the distance between the nozzle and the target decreases, the impact of the collision increases [4]. The proposed method therefore increases the impact generated by the commercially available machine. Moreover, a sufficiently high impact finely destroys the surface of the target [5]. Hence, the system not only washes off the radioactive substances on the surface of the target, but also removes those that have been absorbed into pores on the surface. In other words, the proposed method is used to grind the surface of the target by means of a commercially available high-pressure washing machine. Thus, the method has the ease of use of high-pressure washing and the efficiency of grinding.

Experiments were performed to validate the proposed decontamination method. Figures 1, 2, 3, and 4 shows the results of the experiments, the activities of which were as follows:

- Washing of some types of road materials, e.g., concrete, asphalt and bricks.

- Changing the distance between the washing nozzle and the road.

- Changing the feed rate.

- Washing a $1 \times 1 \mathrm{~m}^{2}$ area entirely.

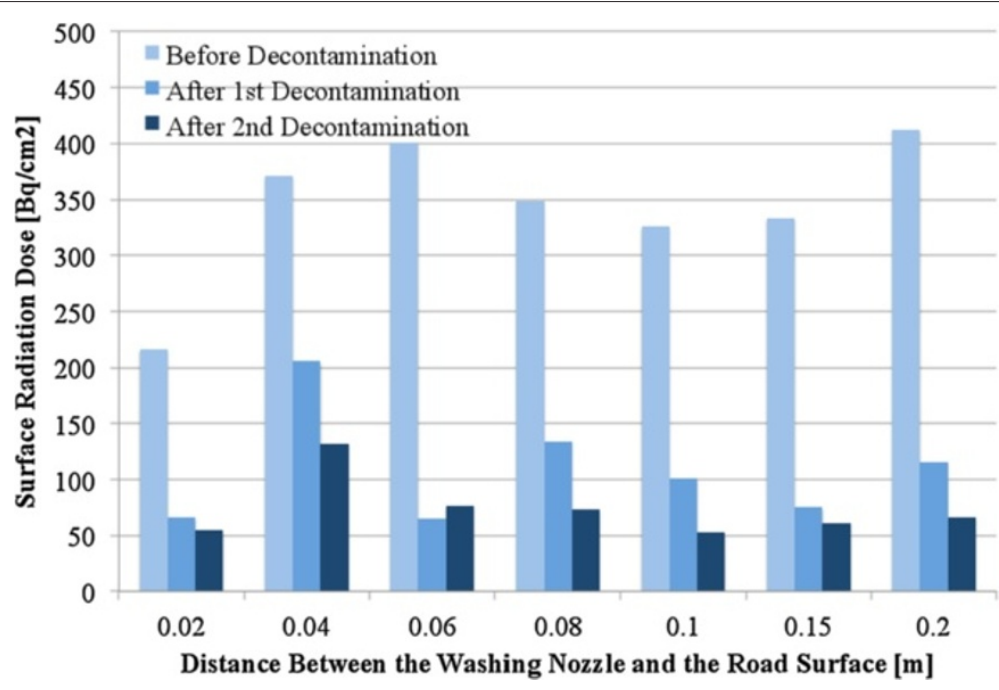

Figure 1 Results of the decontamination experiments - Surface radiation dose with respect to the feed rate and materials on the surface. 


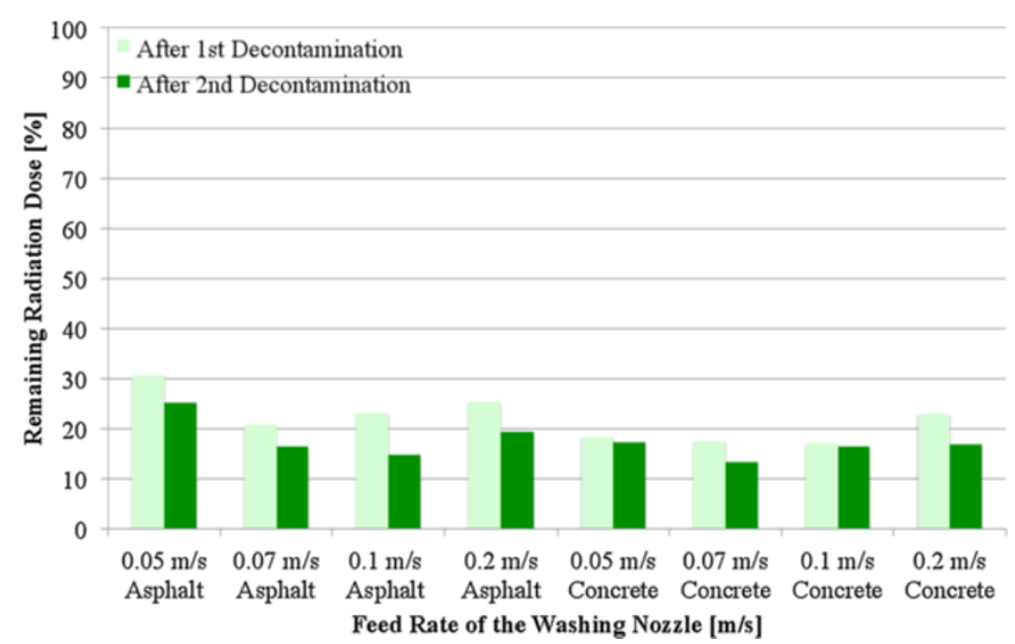

Figure 2 Results of the decontamination experiments - Remaining radiation dose with respect to the feed rate and materials on the surface. When the feed rate was increased, the decontamination efficiency decreased.

- Using a commercially available machine, namely, K4.00 produced by Karchar Co. Ltd., which supplies $7.5 \mathrm{MPa}$ water at $360 \mathrm{l} / \mathrm{h}$.

- Using a Geiger-Mueller survey meter, namely TGS-146B produced by Hitachi Aloka Medical, Ltd. to measure the surface radiation dose before and after the decontamination.

- Measuring the surface radiation dose when the road was completely dry.

The horizontal and vertical axes of the graphs in Figures $1,2,3$, and 4 respectively represent the decontamination time and measured surface radiation dose.
The experimental results revealed the following:

- As the distance between the nozzle and the road decreases, the radiation dose after decontamination also decreases.

- If the nozzle is very close to the road, the road would be destroyed.

- If the nozzle is too far from the road, the efficiency of the decontamination would significantly decrease.

- To be specific, the highest efficiency was recorded when the distance was set to about $10 \mathrm{~mm}$.

- As the decontamination time gets shorter, the efficiency of the decontamination decreases.

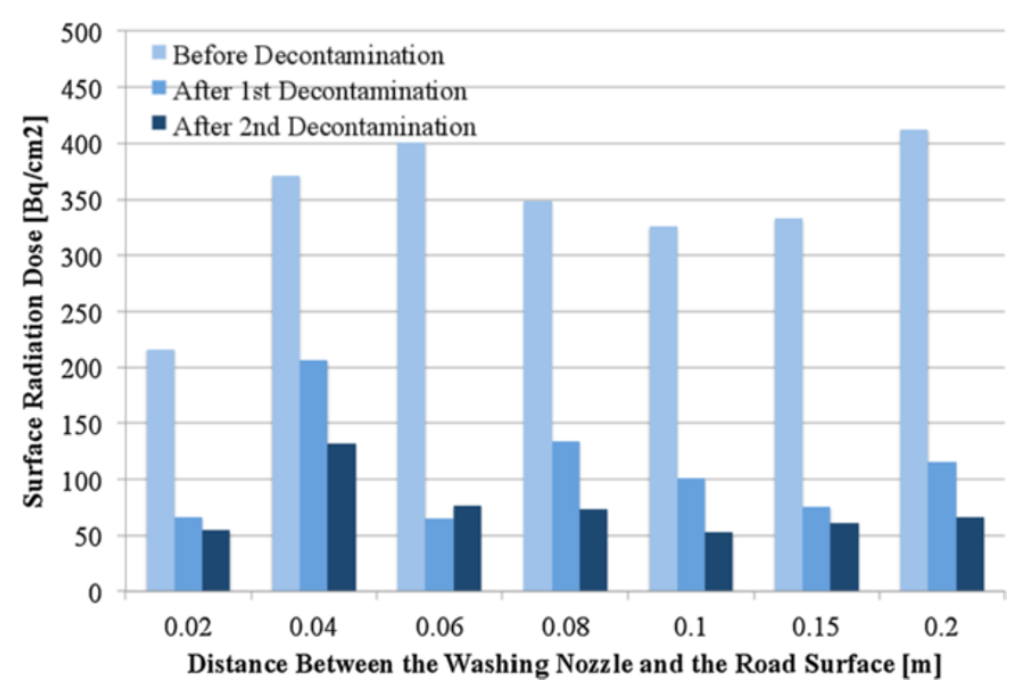

Figure 3 Results of the decontamination experiments - Surface radiation dose with respect to the distance between the ground and the washing nozzle. 


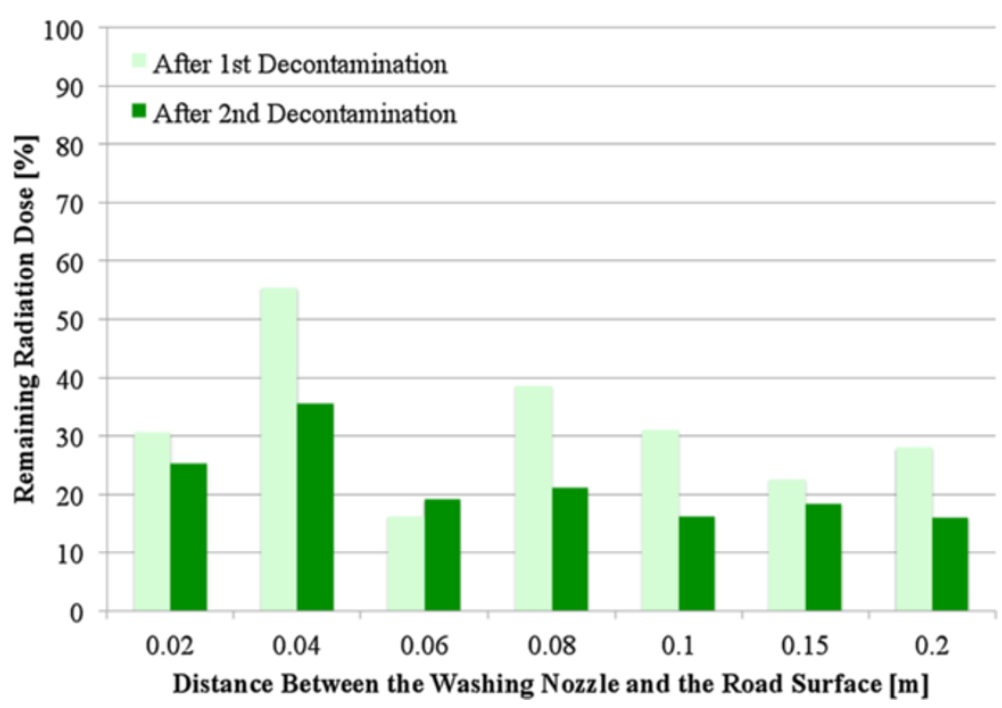

Figure 4 Results of the decontamination experiments - Remaining radiation dose with respect to the distance between the ground and the washing nozzle. Efficient decontamination was achieved by high-pressure washing over short distances. When the distance became too short, the high-pressure water broke the surface of the road.

- The decontamination of an area of $1 \times 1 \mathrm{~m}^{2}$ would require more than $15 \mathrm{~min}$.

Hence, to achieve highly efficient decontamination, a long decontamination time and optimal control of the distance between the nozzle and the road is required.

\section{Washing system comprising multiple coordinated robots}

We propose a decontamination washing system comprising multiple coordinated robots. As mentioned above, the distance between the washing nozzle and the target should be properly controlled. The application of robots is therefore beneficial. As also noted, however, a long washing period is required to achieve sufficient decontamination efficiency. To accelerate the decontamination without decreasing the efficiency, multiple robots working in coordination are employed in the proposed system. Whereas the time required to wash a portion of the road by one robot is not shortened by this system, the time taken to wash the entire road is shortened [6].

Figure 5 illustrates the concept of the proposed system. Multiple transporter robots are used to carry the washing robots from one washing area to another. In addition to the washing and transporter robots, a server robot is used to supply all the washing robots with water for washing the road, and to vacuum the contaminated water from the washing robots. The washing robot is small because it does not include washing water storage, contaminated water storage, high-pressure water pumps, and vacuum pumps. Moreover, the smallness of the washing robot means that the transporter robot is also small.
Figure 6 illustrates the coordination mechanism. The lower part of the figure illustrates the decontamination process using multiple multi-task robots, whereas, for comparison, the upper part illustrates the use of multiple single-task robots. The gray and white frames quantify the area that a washing robot can decontaminate at a particular time. The gray frames represent areas yet to be decontaminated, and the white frames represent areas that have been decontaminated. The blue frames represent the locations of the washing robots. Deep blue indicates areas being washed and light blue indicates areas were washing has been completed. The green frames represent the transporter robots, with deep and light green respectively indicating mobile and stationary ones.

In both cases, every robot shares information about its area, namely, before/after decontamination, impossible to decontaminate (e.g., due to obstacles), and location. After a washing robot has decontaminated its immediate workspace, a transporter robot transports it based on shared information about the state of the different areas. The system uses the shared information to avoid work duplication, thereby achieving high efficiency.

In the case described in the upper part of Figure 6, the transporter robots are able to wait for the washing robots to accomplish their tasks, and their mobile time is shorter than their stationary time. Actually, considering that the time spent on decontaminating a particular area is very long, a long stationary time of the transporter robots is assumed. However, in the case described in the lower part of Figure 6, the transporter robots can transport the washing robots that have finished their decontamination task 


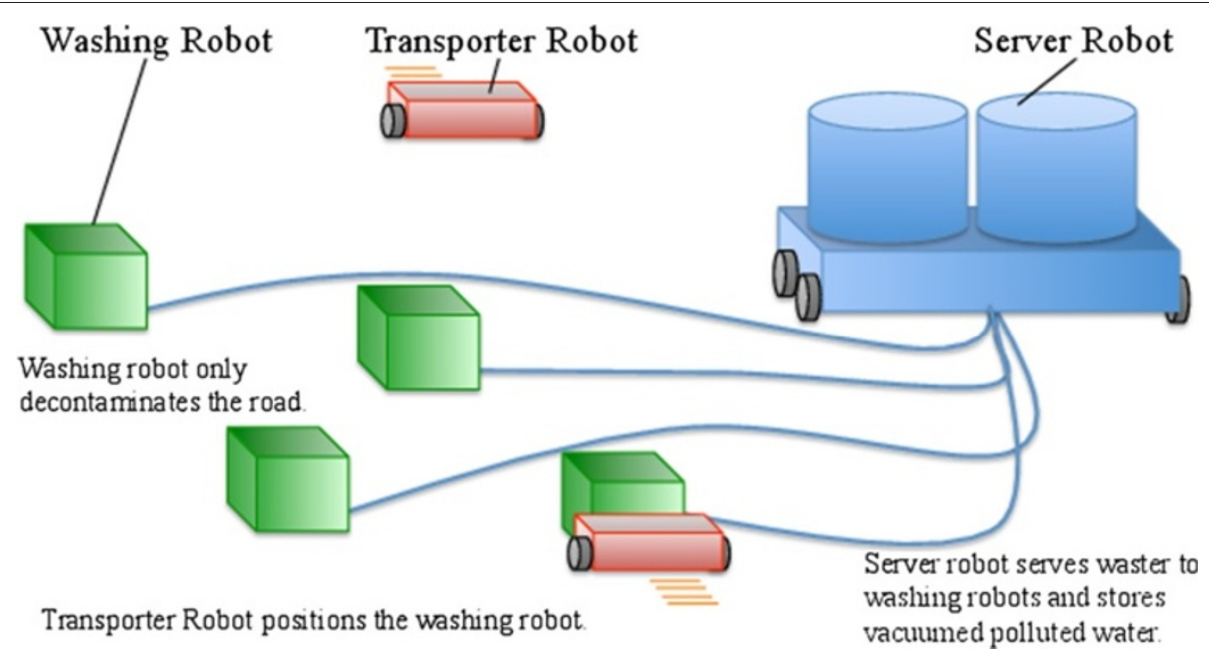

Figure 5 Concept of the complete system. The proposed system comprises three modules: washing robots, transporter robots, and server robot. Multiple washing robots wash the ground. A washing robot spends over $15 \mathrm{~min}$ on each $1 \times 1 \mathrm{~m}^{2}$ area. Multiple transporter robots transport a washing robot. The washing robot does not have a locomotive mechanism because such would amount to wasted resources considering the 15 min spent on washing one location. The server robot is employed to reduce the size of the system. The server robot supplies water to the washing robot and vacuums the polluted water from the washing robot. Compactness is achieved by integrating the pump with the storage of the washing and polluted waters.

individually. Hence, the transporter robots continuously move without time wastage.

\section{Method: Control algorithm for positioning the washing nozzle}

\section{Control algorithm based on a virtual inner model}

The washing robot controls its washing nozzle relative to the target. Specifically, the washing nozzle is initially positioned at a constant velocity in the horizontal direction. The control algorithm, which is based on the virtual inner model with its impedance characteristic, is then used to aim the nozzle in the vertical direction. Based on the algorithm, the robot maintains a distance of $0.01 \mathrm{~m}$ between the washing nozzle and the target. The unevenness and wavy shape of the target is measured by a laser range finder.

Kosuge originally proposed and used the algorithm to control a manipulator relative to the ground based on information obtained by a laser range finder [7]. The algorithm analyzed the normal vectors of the ground using the information obtained by the laser range finder. The manipulator was then controlled to fit the vector of the end-effector to the normal vectors. By this method, he was able to detect land-mines by applying the algorithm to the manipulator, which had a sensor head on its end-effector, and the rough ground.

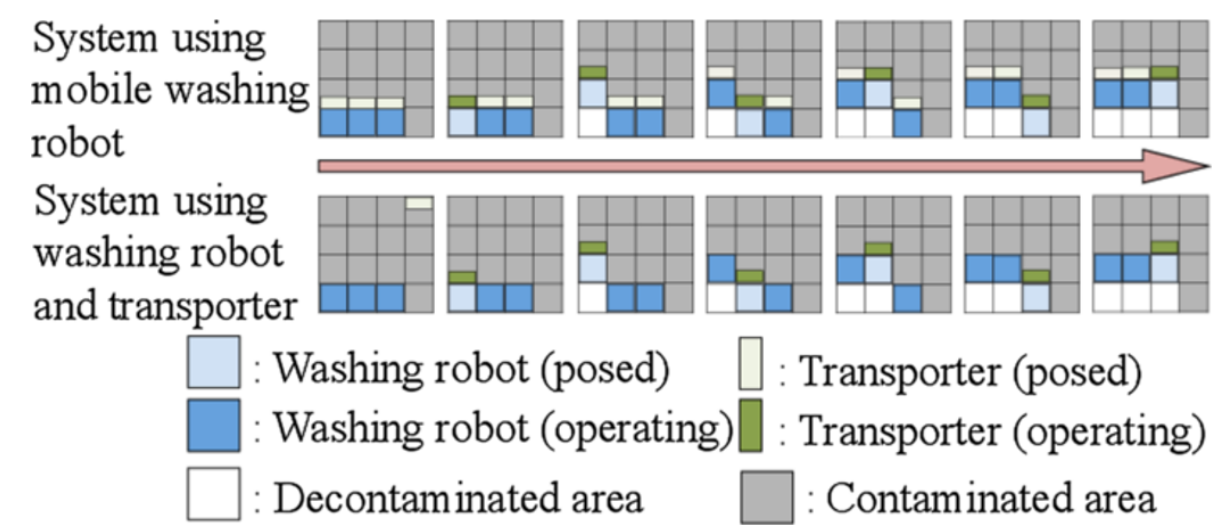

Figure 6 Robot coordination concept of the proposed system. The upper part is a case of decontamination using multiple robots with washing and locomotion mechanisms. The lower part is a case in which multiple washing and transporter robots are employed. The latter case is more efficient. 
In the present study, the target was the road or ground in a residential area. Thus, consideration of a rough ground was not required. It was also unnecessary to consider the direction of the end-effector. The washing robot was controlled relative to the ground using the virtual inner model shown in Figure 7.

The inner virtual model shown in Figure 7 is described by the following equation:

$$
M \ddot{z}(t)=C \dot{\Delta} z(t)+K \Delta z(t)
$$

where $M, C$ and $K$ represent the inertia, viscosity, and elasticity, respectively, and $z(t)$ is the height of the nozzle with reference to the global coordinate system. If $\Delta z(t)$ denotes the difference between the actual nozzle height and the desired nozzle height in the global coordinate system, it can be calculated using the following equation:

$$
\Delta z(t)=z_{d}(t)-z(t)
$$

where $z_{d}(t)$ is the desired nozzle height, which is obtained as follows:

$$
z_{d}(t)=z_{g}(t)+D
$$

where $z_{g}(t)$ is the ground height with reference to the global coordinate system, and $D$ is a constant of the desired distance between the nozzle and the ground.

If $Z(s), \Delta Z(s), Z_{d}(s)$ and $Z_{g}(s)$ respectively represent the Laplace formulations of $z(t), \Delta z(t), z_{d}(t)$ and $z_{g}(t)$, equations (1), (2) and (3) can respectively be transformed as follows:

$$
\begin{aligned}
M s^{2} Z(s) & =C s \Delta Z(s)+K \Delta Z(s) \\
\Delta Z(s) & =Z_{d}(s)-Z(s) \\
Z_{d}(s) & =Z_{g}(s)+\frac{1}{s} D
\end{aligned}
$$

From the above equations, the following is derived:

$$
M s^{2} Z(s)=(C s+K)\left(Z_{g}(s)+\frac{1}{s} D-Z(s)\right)
$$

Equation (7) can be transformed as follows:

$$
Z(s)=\frac{C s+K}{M s^{2}+C s+K} Z_{g}(s)+\frac{C s+K}{M s^{3}+C s^{2}+K s} D
$$

where the first term on the right-hand side of equation (8) is used to adjust the nozzle height $Z(s)$ relative to the ground height $Z_{g}(s)$, and the second term is used to converge the distance between the nozzle and the ground to $D$, which is achieved after sufficient time. Hence, the motion of the nozzle is determined by the first term on the right-hand side of equation (8).

By considering the ground height $Z_{g}(s)$ as an input of the system and the nozzle height $Z(s)$ as an output, the transfer function of the system is derived as follows:

$$
G(s)=\frac{C s+K}{M s^{2}+C s+K}
$$

This transfer function can be expressed as follows:

$$
G(s)=k_{g}\left(1+T_{1} s\right) \frac{1}{1+T_{2} s} \frac{1}{1+T_{3} s}
$$

where the constants are given by

$$
\begin{aligned}
k_{g} & =\frac{1}{2 M K^{2}} \\
T_{1} & =\frac{C}{K} \\
T_{2} & =\frac{C-\sqrt{C^{2}-4 M K}}{2 M} \\
T_{3} & =\frac{C+\sqrt{C^{2}-4 M K}}{2 M s}
\end{aligned}
$$

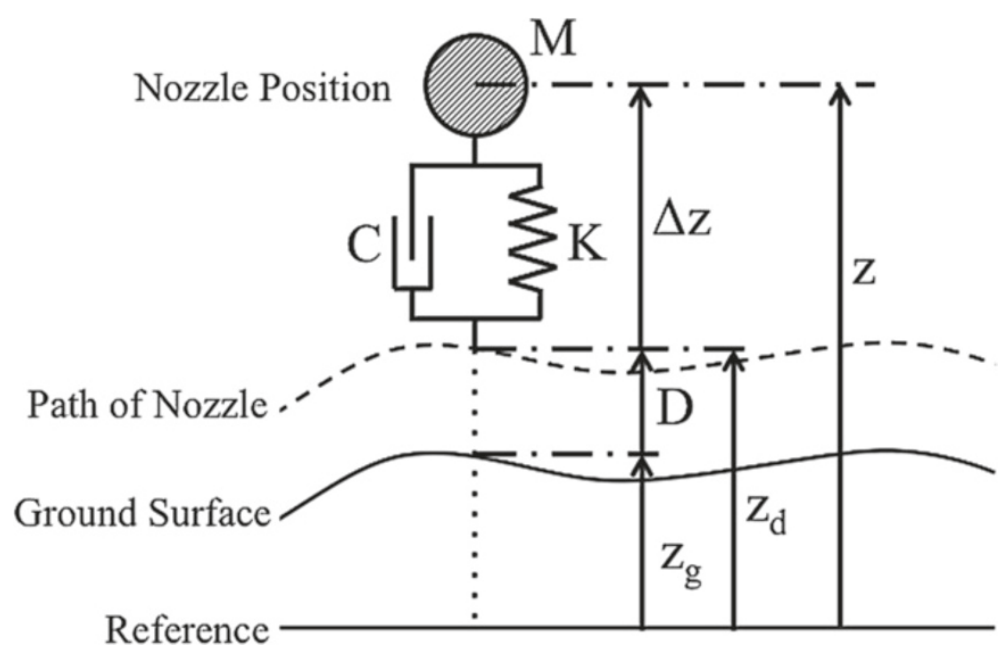

Figure 7 Virtual inner model for controlling the washing robot relative to the ground. The washing nozzle is controlled by means of the virtual inner model using the impedance characteristics. 
Thus, the motion of the system is considered to be a combination of a proportional element, derivative element, and second-order lag element. Figure 8 shows the Bode diagram of the motion, which has corner frequencies of $\frac{1}{T_{1}}, \frac{1}{T_{2}}$ and $\frac{1}{T_{3}}$ based on equation (10).

\section{Adaptive motion of the washing nozzle}

To adapt the motion of the washing nozzle to various ground unevenness, the system analyses the unevenness of the ground along the horizontal path of the washing nozzle using information obtained by the laser range finder. In this study, Fast Fourier Transformation (FFT) was used for the analysis. Based on the frequency characteristics of the FFT, the optimum motion of the washing nozzle was derived.

The laser range finder collected information in each direction. By converting the polar coordinates to Cartesian coordinates, information about the shape of the ground was generated. The shape information consisted of the height of the ground at each horizontal location. Owing to the movement of the washing nozzle at a constant velocity in the horizontal direction, the shape information was transformed to a time function as follows:

$$
z_{g}(t)=z_{g}\left(\frac{P_{t r v}}{V_{t r v}}\right)
$$

where $P_{t r v}$ is the horizontal position of the washing nozzle, and $V_{t r v}$ is its horizontal velocity.

By analyzing the time function of the ground, $z_{g}(t)$, by FFT, the shape information can be quantified for each frequency. Here, $C=2 \sqrt{M K}$ is used for simplify. Hence, the corner frequency of the Bode diagram of the system has one peak for frequency $T_{2}$. By choosing the optimal values

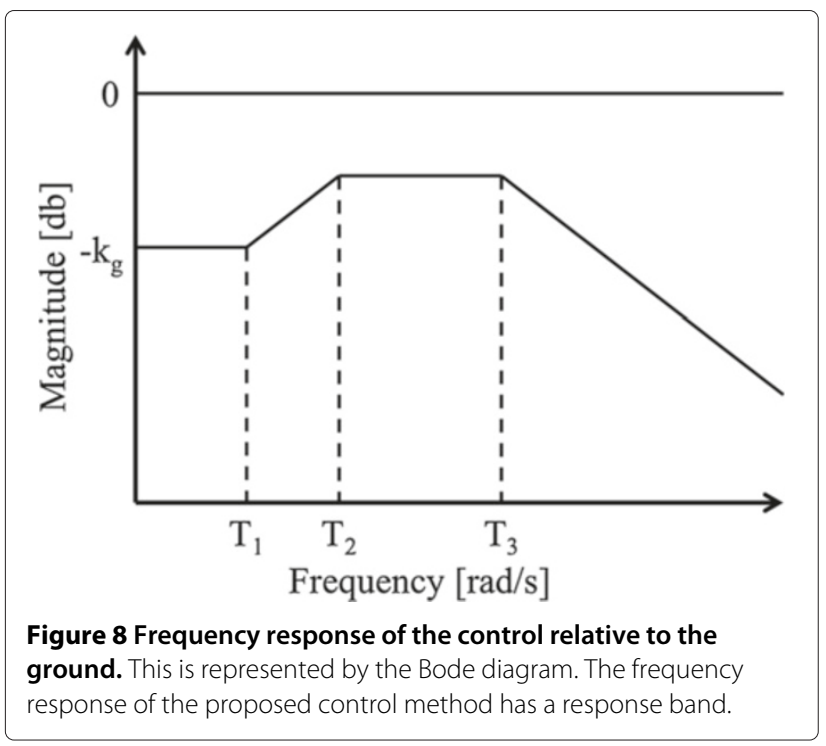

of $M, C$, and $K$, the peak frequency can be accommodated by analyzing the ground using the peak of frequency $T_{2}$.

\section{Result and Discussion}

Implementation of the proposed algorithm

The proposed algorithm was implemented in the washing robot shown in Figure 9. The robot used a laser range finder (URG-04LX, HOKUYO) to measure the unevenness of the ground, and a high-pressure washing system (K4.00, Karcher) for the decontamination. The washing nozzle of the system was positioned with $3 \mathrm{DoF}$ in the X$\mathrm{Y}-\mathrm{Z}$ directions of an XYZ stage on which the robot was installed.

For the decontamination, the washing robot first measured the unevenness of the ground using the laser range finder. Specifically, when the $\mathrm{x}$-axis was set toward the front of the robot and the $\mathrm{z}$-axis was set in the vertical direction, the robot measured the two-dimensional unevenness of the ground in the $y-z$ plane. Based on the obtained information, the robot controlled the washing nozzle at a constant velocity in the y-direction, and used the proposed algorithm to control it in the $\mathrm{z}$-direction. By this means, the washing robot moved the washing nozzle relative to the ground.

When the robot measured the unevenness of the ground from a single position, there was occlusion. To avoid this, the measurements were taken from multiple positions and combined. Figure 10 shows an instance. As shown in the upper left figure, the robot measures the unevenness of the ground using a laser range finder attached to the washing nozzle. In the upper right figure, the robot measures the ground from a single position and the opposite side of the unevenness is occluded from the sensor. Thus, there

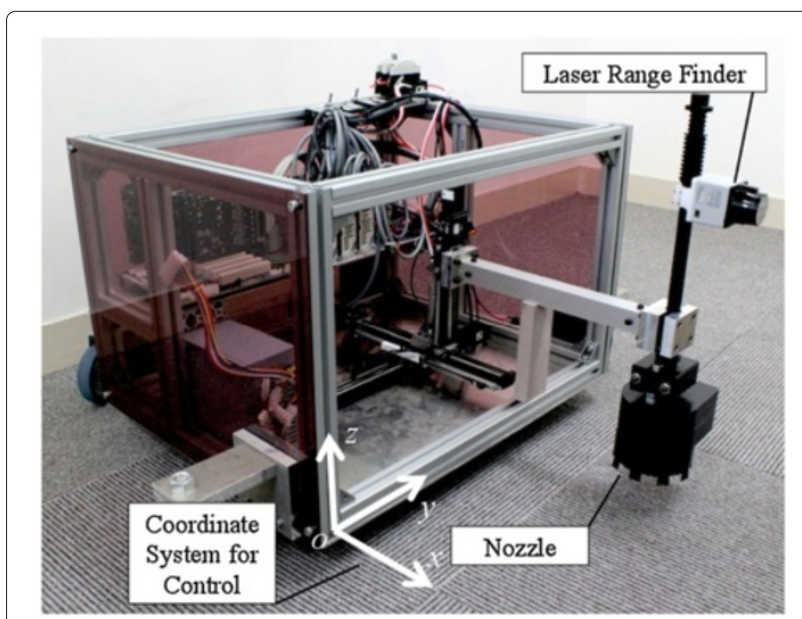

Figure 9 Experimental washing robot system. The washing robot does not have a locomotion mechanism. It has a laser range finder, a washing nozzle, and an $x-y-z$ stage for positioning the laser range finder and the nozzle. 


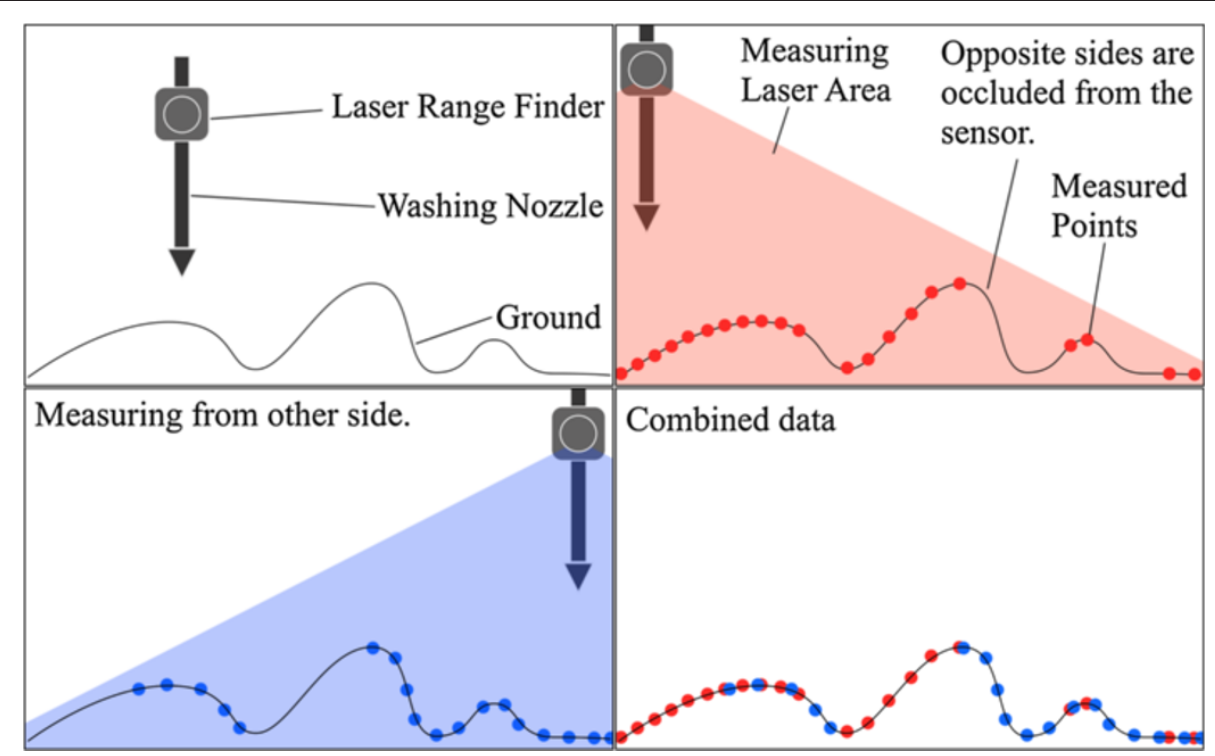

Figure 10 Concept of the measurement of the unevenness of the ground from multiple points. The robot measures the unevenness of the ground using a laser range finder attached on the washing nozzle. In the upper right figure, the robot measures the ground from a single position, and the opposite side of the unevenness is occluded from the sensor. To obtain information about the opposite side, as shown in the lower left figure, the robot takes the measurement from another position. By combining the measurement data obtained from the two positions, the complete shape of the ground can be determined.

is no information about the opposite side. To obtain information about the opposite side, as shown in the lower left figure, the robot takes the measurement from another position. By combining the measurement data obtained from the two positions, the complete shape of the ground can be determined.

If $N$ represents the number of data obtained by a single scan of the laser range finder, and $n$ represents the number of scans, the ground unevenness information obtained by the laser range finder can be expressed as follows:

$$
\begin{aligned}
P_{U} & =\left\{p_{U(i)}\right\}(i=1,2,3 \cdots n N) \\
p_{U(i)} & =\left[y_{U(i)} z_{U(i)}\right]^{T}
\end{aligned}
$$

where $p_{U(i)}$ are arranged in ascending order.

The measured points $P_{U}$ are discontinuous. To generate continuous ground information, $z_{g}(t)$ was calculated on $\left[y_{U(i)}, y_{U(i+1)}\right)$ using the following equation, where $y(t)$ is the $y$-direction position of the washing nozzle:

$$
z_{g}(t)=\frac{z_{U(i+1)}-z_{U(i)}}{y_{U(i+1)}-y_{U(i)}}\left(y(t)-y_{U(i)}\right)+z_{U(i)}
$$

Using $z_{g}(t)$, the desired position of the washing nozzle in the $\mathrm{z}$-direction is generated based on equation (8). To be specific, equation (8) was discretized by bilinear transform as follows:

$$
z_{i}=\sum_{j=1}^{3} \frac{k_{z j}}{k_{z 0}} z_{i-j}+\sum_{j=0}^{3} \frac{k_{g j}}{k_{z 0}} z_{g i-j}+\frac{k_{D}}{k_{z 0}} D
$$

where $z_{i}$ is the desired height of the nozzle on the $i$ th control loop and $z_{g i}$ is the ground height calculated by equation (18) on the $i$ th control loop. The coefficients in equation (19) are as follows:

$$
\begin{aligned}
k_{z 0} & =4 M+2 C T+K T^{2} \\
k_{z 1} & =12 M+2 C T-K T^{2} \\
k_{z 2} & =-12 M+2 C T+K T^{2} \\
k_{z 3} & =4 M-2 C T-K T^{2} \\
k_{g 0} & =2 C T+K T^{2} \\
k_{g 1} & =-2 C T+K T^{2} \\
k_{g 2} & =-2 C T-K T^{2} \\
k_{g 3} & =2 C T-K T^{2} \\
k_{D} & =4 K T^{3}
\end{aligned}
$$

where, $T$ is the control periodic time.

The nozzle height is controlled on each control loop by the following control algorithm:

$$
\tau_{i}=\frac{K_{p}}{L}\left(z_{i}-z_{c i}\right)+\frac{K_{d}}{L}\left(\dot{z}_{i}-\dot{z}_{c i}\right)
$$

where $\tau_{i}$ is the torque of a motor attached to a ball screw and used to adjust the height of the washing nozzle. $z_{c i}$ is the current height of the washing nozzle calculated by an encoder attached to the ball screw. 


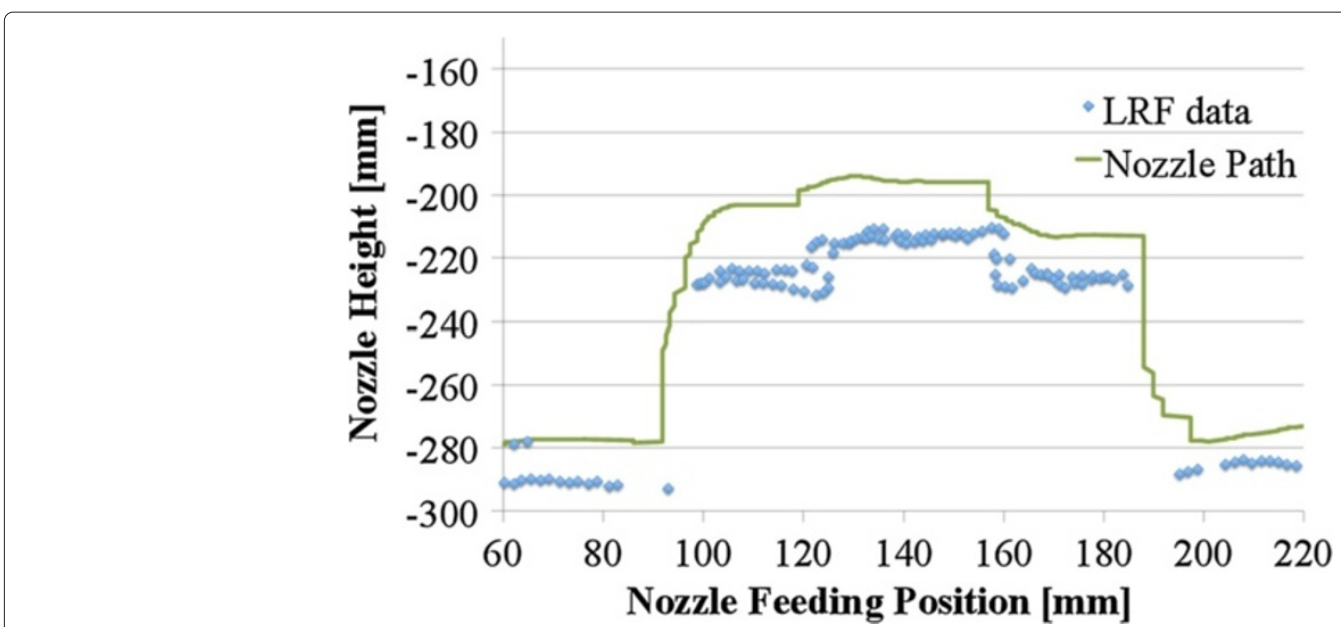

Figure 11 Nozzle trajectory during the control experiment. The solid line indicates the actual trajectory of the washing nozzle. The broken line represents the sensor information obtained by the laser range finder. The solid line agrees well with the broken line.

\section{Experiment of control the washing nozzle following the ground}

The proposed system was experimentally validated using square pillars laid on the ground to imitate unevenness. The washing robot used the laser range finder to measure the ground and pillars from two positions, namely, the beginning and finishing points of the decontamination. The robot controlled the washing nozzle at $5 \mathrm{~mm} / \mathrm{s}$ in the y-direction and by the proposed algorithm in the $\mathrm{z}$-direction. The parameters of the virtual inner model were determined using the method described in Section 'Adaptive motion of the washing nozzle'. Specifically, by setting $M=10$ for simplicity in this case, the parameters were obtained as $C=10$ and $K=2.5$. The control periodic time $T$ was set to 0.001 .

Figure 11 shows the information obtained by the laser range finder and the path of the washing nozzle. The abscissa and ordinate of the graph respectively represent the positions in the $y$ - and z-direction. The dots represent the data obtained by the laser range finder, and the curved line represents the path of the washing nozzle. As shown in the figure, the washing nozzle moved relative to the ground based on information obtained by the laser range finder. The path of the washing nozzle was, however, not stable, which was because the precision of the laser range finder was $\pm 10 \mathrm{~mm}$. The information of the laser range finder therefore contained noise. Figure 12 shows a series of photographs of the experiment.

\section{Decontamination experiment}

A washing nozzle that could vacuum the contaminated water was installed for the experiment. The GM survey meter TGS-146B (Hitachi Aloka Medical, Ltd.) was used to measure the surface radiation dose when the road was completely dry before and after decontamination. Figure 13 shows the conditions of the decontamination experiment. As indicated, a single area was used for the experiment, which included the actual experimen-

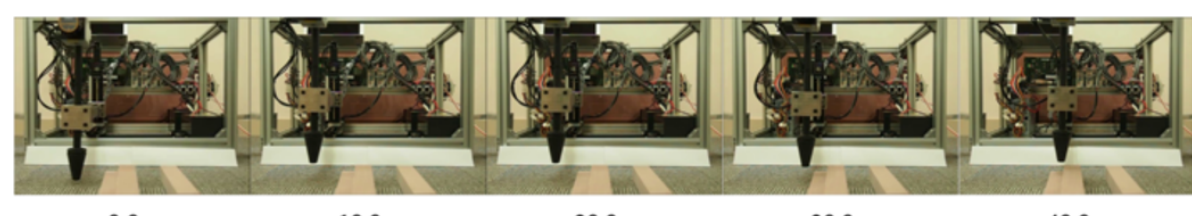

$0.0 \mathrm{~s}$

$10.0 \mathrm{~s}$

$20.0 \mathrm{~s}$

$30.0 \mathrm{~s}$

$40.0 \mathrm{~s}$

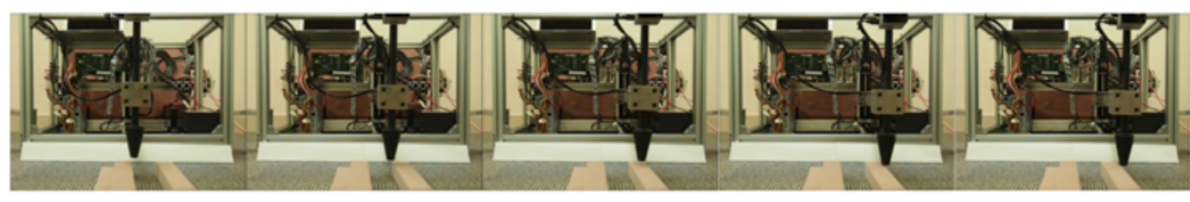

$50.0 \mathrm{~s}$

$60.0 \mathrm{~s}$

$70.0 \mathrm{~s}$

$80.0 \mathrm{~s}$

$90.0 \mathrm{~s}$

Figure 12 Photographs of the control experiment. The sequence photographs show the trajectory of the washing nozzle during the control experiment. 


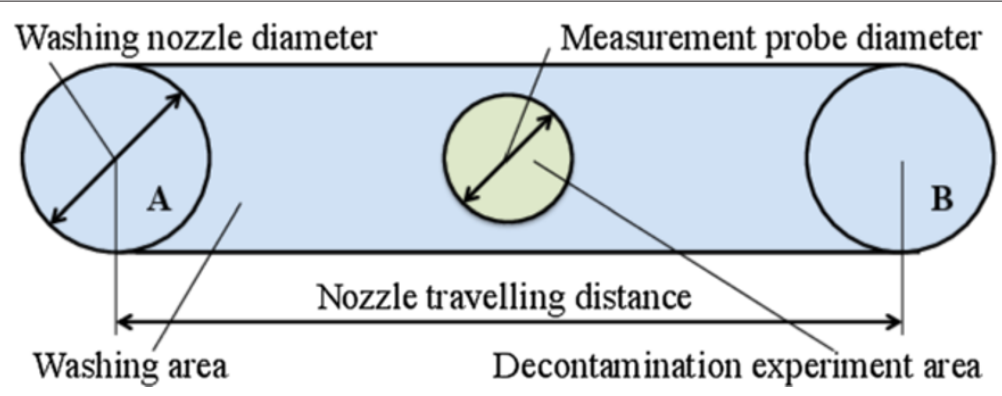

Figure 13 Conditions of the decontamination experiment. A single area was used for the experiment, and included the actual experimental area decontaminated by the system. The radius of the washing nozzle was larger than that of the probe. The beginning and finishing points of the decontamination experiment were therefore respectively on the left and right side of the experimental area.

tal area decontaminated by the system. The radius of the washing nozzle was larger than that of the GM survey meter probe. The beginning and finishing points of the decontamination experiment were therefore respectively on the left and right side of the experimental area.

The experiment was performed at three different locations in Koriyama, Fukushima, Japan. All the experimental areas were asphalt pavements that had been previously decontaminated. The surface radiation doses were therefore sufficiently lower than those of un-decontaminated areas. The previous decontaminations had been done by high-pressure washing. As explained earlier, although the radioactive substances had been removed from the surface of the experimental areas, they were still present in the pores.

Figure 14 shows the washing robot during the experiment, with Figure 15 focusing on the washing nozzle. The distance between the washing nozzle and the ground was set at $10 \mathrm{~mm}$, and the feed rate of the washing nozzle at 5 $\mathrm{mm} / \mathrm{s}$. Under these conditions, the washing robot decontaminated an area with a width equal to the radius of the nozzle, which was $100 \mathrm{~mm}$, and a length of $200 \mathrm{~mm}$. The

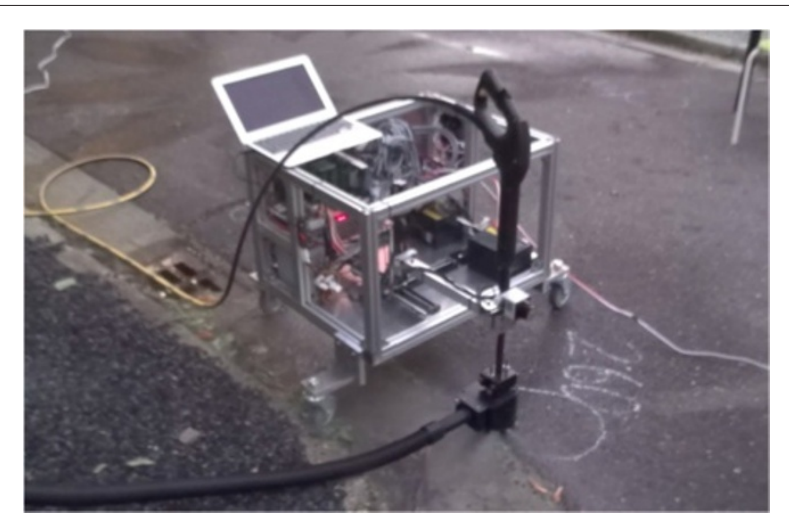

Figure 14 The robot during the decontamination experiment General view. parameters of the virtual inner model were approximately $M=0.5, C=40$, and $K=800$. The control periodic time $T$ was set to 0.001 .

The trajectory of the washing nozzle during the experiment is shown in Figure 16. The horizontal axis represents the time from the beginning of the experiment. The first vertical axis represents the height of the washing nozzle, and the red and green lines respectively represent the desired height and actual height of the washing nozzle. The lines show that the nozzle properly followed the desired height. The second vertical axis represents the error between the desired and actual heights of the washing nozzle. Immediately after the beginning of the experiment, the error was greater than $0.6 \mathrm{~mm}$; however, after a few seconds, the nozzle followed the desired trajectory well and the error became less than $0.1 \mathrm{~mm}$. These results indicate that the effects of the reactive force of the high pressure water jet and the adhesive force of the vacuuming of the polluted water on the control system of the washing nozzle are negligible.

The results of the experiments are given in Table 1. Before the decontamination, the surface radiation dose of each point was between 70 and $200 \mathrm{~Bq} / \mathrm{cm}^{2}$. It

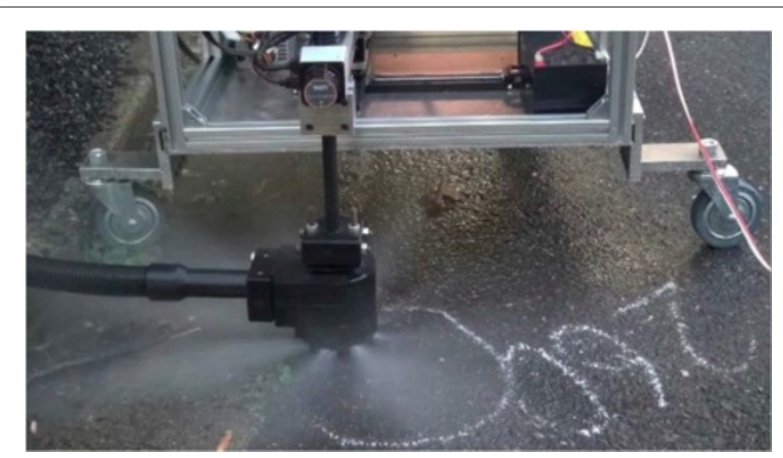

Figure 15 The robot during the decontamination experiment Around the Nozzle. Substantial water leakage is shown, which was because the positioning precision was degraded by sensor jamming due to the water leakage. 


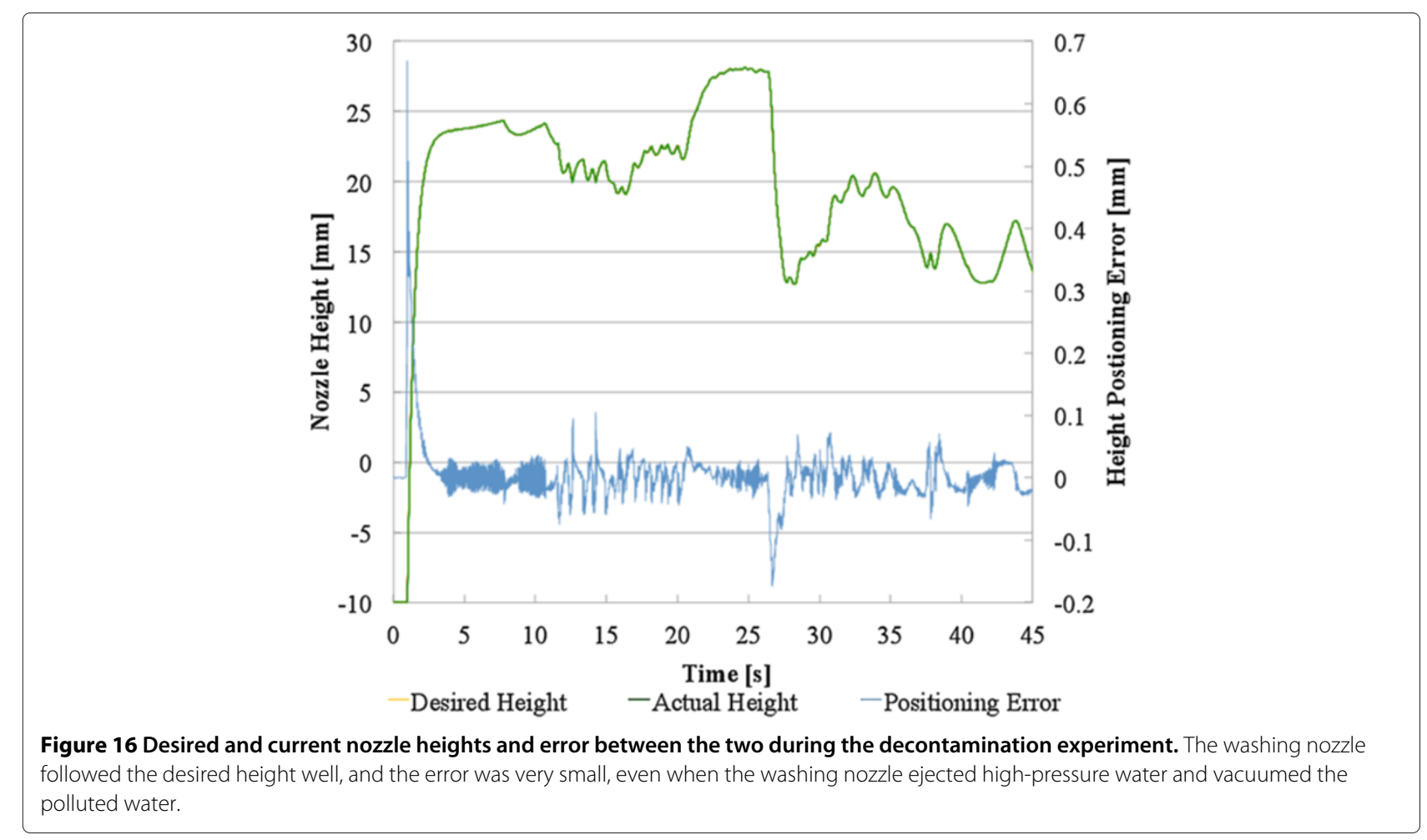

was found that almost all the remaining contaminants were cesium 134 and 137 , and the ratio of cesium 134 to cesium 137 was 2:3. The relationship between the surface radiation dose and the air radiation dose for cesium 134 is $1 \mathrm{~Bq} / \mathrm{cm}^{2}=5.4 \times 10^{-2} \mu \mathrm{Sv} / \mathrm{h}$, and that for cesium 137 is $1 \mathrm{~Bq} / \mathrm{cm}^{2}=2.1 \times 10^{-2} \mu \mathrm{Sv} / \mathrm{h}$. Regarding the ratio between cesium 134 and 137, the relationship is $1 \mathrm{~Bq} / \mathrm{cm}^{2}=3.42 \times 10^{-2} \mu \mathrm{Sv} / \mathrm{h}$. Thus, the air radiation dose of each point was estimated to be between 0.69 and $1.97 \mu \mathrm{Sv} / \mathrm{h}$. Those of two points were slightly below 1 $\mathrm{mSv} /$ year, which is the standard threshold specified by the Ministry of the Environment. The air radiation dose of the first point was above the threshold despite that the point had been previously decontaminated. It can be seen from the table that the proposed system further reduced the surface radiation dose. As previously explained, the system removed the radioactive substances not only from the surface, but also from the pores on the surface. The experimental results thus validated the efficacy of the proposed system.

Table 1 Decontamination results

\begin{tabular}{ccc}
\hline Area & Before $\left[\mathbf{B q} / \mathbf{c m}^{\mathbf{2}}\right]$ & After $\left[\mathbf{B q} / \mathbf{c m}^{\mathbf{2}}\right]$ \\
\hline A & 200 & 129 \\
\hline B & 70 & 55 \\
\hline C & 95 & 60 \\
\hline
\end{tabular}

Before the previous decontamination, the road surface had an average dose of $400 \mathrm{~Bq} / \mathrm{cm}^{2}$. The efficiency of the previous decontamination was thus about $50 \%$ to $70 \%$. Based on this, the efficiency of the proposed system is estimated to be about $80 \%$. The system proposed by TOSHIBA Co. has an efficiency of $90 \%$ using dry-ice blast, and that proposed by Muramoto Co. has an efficiency of $80 \%$ using super-high-pressure water (pressure above 100 $\mathrm{MPa}$ ). The proposed system has an efficiency equivalent to those of other conventional methods that utilize special and large equipment, and this is achieved by commercially available machines.

The washing nozzle used in this study could vacuum almost all the contaminated water when adhered to the ground. However, as in the experiment described in the previous section, the movement of the nozzle was affected by the noise that resulted from the precision of the laser range finder. Hence, water leaked through the gap between the nozzle and the ground and wetted the surrounding un-decontaminated area. Because the laser range finder was one of the photo-sensors, the measured data of the wet and dry target areas were different. The water leakage therefore made decontamination by the proposed system difficult.

\section{Conclusions}

In this paper, we proposed a high-pressure washing system for use in the decontamination of radioactive 
substances spread by the accident that occurred at the TEPCO Fukushima 1st Nuclear Power Plant. The proposal was prompted by the unsatisfactory progress of decontamination workers in residential areas. The proposed system, which is targeted at accelerating the decontamination activities, consists of multiple washing and transporter robots. The washing robots execute highly efficient decontamination using commercially available high-pressure washing equipment, and work in coordination with the transporter robots to shorten the decontamination time. The concept of the proposed system and the control algorithm of the high-pressure washing nozzle were described in this paper, as well as the experimental validation of the proposed system. The proposed system and method cannot only be utilized in residential areas, but also in high-radiation-dose areas such as around a brown nuclear reactor. Furthermore, a worker can transport the washing robot for its separate use. In a future work, we would demonstrate the proposed system using a nozzle control that is not based on photosensors, and also propose ways for accelerating decontamination activities by industry-academia-government collaboration.

\section{Competing interests}

The authors have declared that no competing interests exist.

\section{Authors' contributions}

MiE constructed the proposed algorithm, designed the washing robot, implemented the algorithm and drafted the manuscript. MaE did the decontamination efficiency experiment for discovering the decontamination characteristics and the decontamination experiment by using the robot. TK conceived of the study and participated in its design and coordination and helped to draft the manuscript. All authors read and approved the final manuscript.

\section{Acknowledgements}

This research was partially supported by the Japan Science and Technology Agency, Adaptable and Seamless Technology transfer Program through target driven R\&D, Feasibility Stage, 2011-2013 (232Z02315B, 241FT0287, Mitsuru Endo) and the Collage of Engineering, Nihon University, Research Funds of the Collage of Engineering, 2013 (Mitsuru Endo).

Received: 25 January 2014 Accepted: 4 July 2014

Published online: 02 September 2014

\section{References}

1. Record and proposal about robots developed for nuclear reactor accident. The Robotics Society of Japan, East Japan earthquake disaster connection Committee. In Japanese

2. Nishimura K, Koyanagi E, Yoshida T (2012) Improvement and survey about quince for nuclear power plant accident. 30th Congress of the Robotics Society of japan. In Japanese

3. Horikome A, Yamada H, Hirose S, Endo G, Fukushima F (2013) Development of the interference wire drive model multi-joints arm. 31th Congress of the Robotics Society of japan. In Japanese

4. Shimizu S, Shinoda Y, Peng G (2008) Flow characteristics of water jet issuing from a fan jet nozzle. Water Jetting BHR Group

5. Shimizu S, Hiroaki H (2006) Flow structure and erosive characteristics of water jet issuing from a fan jet nozzle. Water Jetting BHR Group: 337-345
6. Endo M, Kakizaki T (2013) Road surface washing system for decomtaminating radioactive substances using multiple mobile robots. In: Proceedings of the 2013 JSME Conference on Robotics and Mechatronics, pp 1-204

7. Yabushita H, Hirata Y, Kosuge K (2004) Small reaction manipulator for gpr sensing head maneuver. In: Selected Papers of the 1st Int. Symposium on System and Human Science (SSR2003), p 18

doi:10.1186/s40648-014-0013-8

Cite this article as: Endo et al.: Washing operation of a road surface washing mechanism for decontaminating radioactive substances. ROBOMECH Journal 2014 1:13

\section{Submit your manuscript to a SpringerOpen ${ }^{\circ}$ journal and benefit from:}

- Convenient online submission

Rigorous peer review

- Immediate publication on acceptance

- Open access: articles freely available online

- High visibility within the field

- Retaining the copyright to your article

Submit your next manuscript at $\gg$ springeropen.com 